\title{
Upgrade of the ALICE Inner Tracking System
}

\author{
Iouri Belikov ${ }^{1, \star}$ for the ALICE Collaboration \\ ${ }^{1}$ IPHC, Université de Strasbourg, CNRS-IN2P3, 23 rue du Loess, BP28, 67037 Strasbourg cedex 2
}

\begin{abstract}
A Large Ion Collider Experiment (ALICE) is built to study the properties of the strongly interacting matter created in heavy-ion collisions at the LHC. With the upgrade of its Inner Tracking System (ITS), the ALICE experiment is going to increase the rate of data taking by almost two orders of magnitude. At the same time, the precision of secondary vertex reconstruction will become by at least a factor 3 better than it currently is. In this talk, we briefly show some selected physics results motivating the upgrade of the ITS, describe the design goals and the layout of the new detector, and highlight a few important measurements that will be realized after the completion of this upgrade.
\end{abstract}

\section{ALICE experiment at CERN}

A Large Ion Collider Experiment (ALICE) [1-3] is one of the four major experiments currently operating on the beams of the Large Hadron Collider (LHC) at the European Organization for Nuclear Research (CERN). The aim of this experiment is to study the physics of strongly interacting matter at extreme energy densities, where the formation of a new phase of matter, the quark-gluon plasma, is expected. For this purpose, the collaboration is carrying out series of comprehensive measurements of hadrons, electrons, muons and photons produced in the collisions of heavy nuclei. In addition, ALICE is studying proton-proton interactions both as a reference for the heavy-ion collisions and also in physics areas where ALICE is competitive with other LHC experiments.

\section{Selected (Run I) results and their limitations}

An important part of the ALICE physics program is dedicated to high precision measurements of charm and beauty production in heavy-ion collisions. The ultimate goals of the heavy-flavour studies include:

- Thermalization of heavy quarks in the produced medium (by measuring baryon-to-meson ratios for charm and beauty particles, ratios of yields of heavy-flavour particles with strange content, and azimuthal flow anisotropy for as many as possible heavy-flavour species).

- Parton mass and colour-charge dependence of in-medium energy losses (by measuring the momentum-dependent nuclear modification factors for B and D mesons, and comparing them with those for light-flavour particles).

^e-mail: iouri.belikov@iphc.cnrs.fr 
Using the data recorded during the LHC Run I (2009-2013), the ALICE Collaboration has already published several important results on the $\mathrm{D}$-meson production in $\mathrm{Pb}-\mathrm{Pb}$ collisions. For example, the left panel of Figure 1 shows the $p_{\mathrm{T}}$-dependent nuclear modification factor for D-mesons (average of $\mathrm{D}^{0}, \mathrm{D}^{+}$and $\mathrm{D}^{*+}$ ) compared to that of pions and charged particles in the $0-10 \%$ centrality class. There is an indication that production of $\mathrm{D}$-mesons in $\mathrm{Pb}-\mathrm{Pb}$ collisions at lower $p_{\mathrm{T}}$ is less suppressed than that of light-flavour particles. The ALICE measurement of the second-order azimuthal flow harmonic $v_{2}$ is presented on the right panel of the Figure. The data suggest that the $v_{2}$ for D-mesons is compatible with that of charged particles and is larger than 0 over a wide momentum range. But, in both cases, the current statistical and systematic uncertainties do not allow for firm conclusions. In addition, the read-out rate capabilities and space-point precision of the present Inner Tracking System (ITS) are not sufficient to perform similar measurements with beauty particles (B-mesons), which are important to prove the existence of quark-mass ordering: $R_{\mathrm{AA}}^{\mathrm{B}}>R_{\mathrm{AA}}^{\mathrm{D}}>R_{\mathrm{AA}}^{\text {charged }}$ and $v_{2}^{\mathrm{B}}<v_{2}^{\mathrm{D}}<v_{2}^{\text {charged }}$.
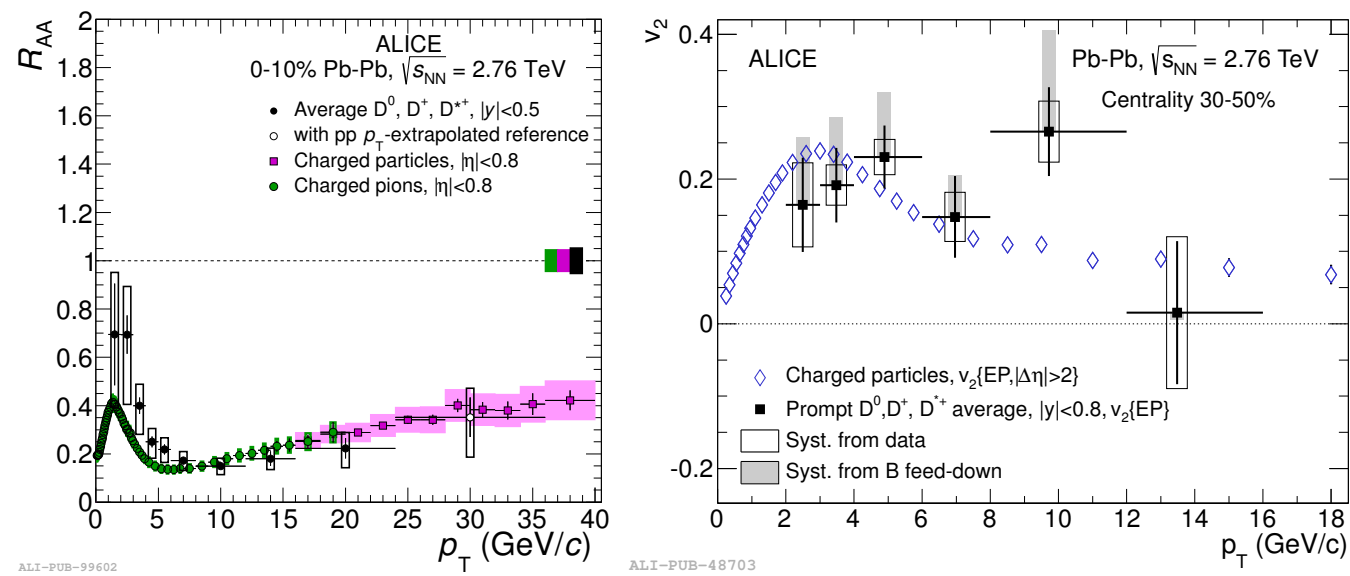

Figure 1. Left panel: Nuclear modification factor $R_{\mathrm{AA}}$ for D-mesons, pions and charged particles [4]. Right panel: Amplitude of the second-order flow harmonic $v_{2}$ for D-mesons and charged particles [5]. Statistical and systematic uncertainties are represented by bars and boxes respectively.

At the same time, even though ALICE has already measured several baryon-to-meson ratios with light-flavour particles [6,7], the extension of these results towards the heavy-flavous sector is currently not possible. The read-out rates and precision of track reconstruction provided by the present ITS do not allow for detecting the heavy-flavous baryons $\left(\Lambda_{c}\right.$ and $\left.\Lambda_{b}\right)$ in high-track-multiplicity environment of $\mathrm{Pb}-\mathrm{Pb}$ collisions at the $\mathrm{LHC}$.

\section{Upgrade of the Inner Tracking System}

The limitations of the present ITS will be radically reduced with the planned upgrade [8]. The general layout of the upgraded ITS is shown in Figure 2. The detector will be $1.5 \mathrm{~m}$ long, with the outer radius of $40 \mathrm{~cm}$, and the overall surface of about $10 \mathrm{~m}^{2}$ will be covered with silicon pixel sensors. This will be a "12.6 Giga-pixel camera", with an estimated cost of about 13.6 million Swiss Francs.

The detector will consist of seven layers, with the first layer as close to the interaction point as $22.4 \mathrm{~mm}$. The material budget of the three innermost layers will be as low as $0.3 \%$ of a radiation length. Combined with the space-point precision of about $5 \mu \mathrm{m}$, the expected track impact-parameter 


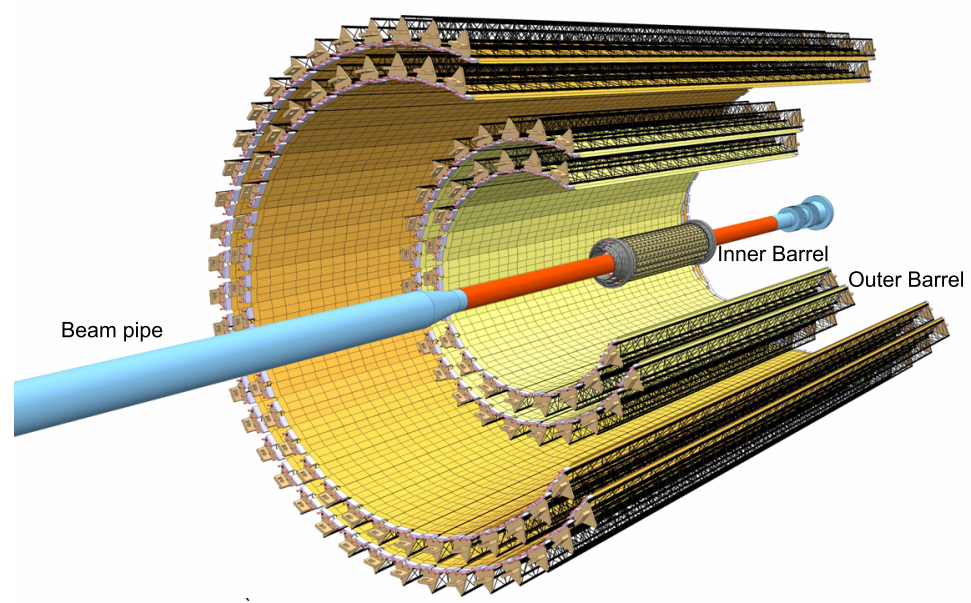

Figure 2. The general layout of the upgraded ITS detector.

resolution at $p_{\mathrm{T}} \sim 1 \mathrm{GeV} / c$ will be about $20 \mu \mathrm{m}$, which is a factor 3 (in the transverse plane) and 5 (in the beam direction) better as compared with the current situation.

The new detector will also allow for an efficient track reconstruction down to very low $p_{\mathrm{T}}$. The simulations show that the tracking efficiency at $p_{\mathrm{T}} \sim 0.1 \mathrm{GeV} / c$ will be a factor about 6 higher than it is now. This will be of a great importance for planned di-electron measurements (see Table 1) and the detection of $\mathrm{D}^{*}$ and $\mathrm{D}_{\mathrm{S}}$ mesons.

All seven layers of the upgraded ITS will be equipped with the ALPIDE chip [8]. Taking advantages of the $0.18 \mu \mathrm{m}$ CMOS technology by TowerJazz [9], this chip combines the sensitive part and the read-out electronics within the same piece of silicon. The main characteristics of the chip are:

- overall chip size: $15 \times 30 \mathrm{~mm}^{2}$;

- pixel size: $29 \times 27 \mu \mathrm{m}^{2}$;

- detection efficiency: $>99 \%$, with a noise probability of $<10^{-6}$;

- time resolution: about $2 \mu \mathrm{s}$;

- in-pixel discriminators and in-matrix address encoder with asynchronous sparsified readout;

- power consumption: $40 \mathrm{~mW} / \mathrm{cm}^{2}$;

- high radiation tolerance: tested up to $2.7 \mathrm{Mrad}$ (TID), and $1.7 \times 10^{13} 1 \mathrm{MeV} \mathrm{n}_{\mathrm{eq}} / \mathrm{cm}^{2}$ (NIEL)

The upgraded ITS will be almost two orders of magnitude faster than now. The read-out electronics of the detector will be able to register events with a typical rate of $50 \mathrm{kHz}$ and a few $100 \mathrm{kHz}$ for minimum bias $\mathrm{Pb}-\mathrm{Pb}$ and $\mathrm{pp}$ collisions respectively.

The parts of the detector will have to be aligned with the precision of better than $5 \mu \mathrm{m}$ by means of dedicated software. The data coming out of the detector will be reconstructed quasi-online, using a dedicated Online-Offline $\left(\mathrm{O}^{2}\right)$ computer farm [10].

Overall, the upgrade of the Inner Tracking System will be accomplished by the end of the LHC Long Shutdown 2 (2021). 


\section{Physics with the upgraded ITS}

The expected physics reach for various observables is summarized in Table 1, in terms of minimum accessible $p_{\mathrm{T}}$ and of statistical uncertainties. We consider a scenario with an integrated luminosity of $10 \mathrm{nb}^{-1}$, fully used for minimum-bias $\mathrm{Pb}-\mathrm{Pb}$ data collection, and a low-magnetic-field run with $3 \mathrm{nb}^{-1}$ of integrated luminosity for the low-mass di-electron studies. The case of the programme up to Long Shutdown 2 is shown for comparison. In this case, a delivered luminosity of $1 \mathrm{nb}^{-1}$ is assumed, out of which $10 \%$ is recorded with a minimum-bias trigger.

In the upgrade case, the systematic uncertainties will be reduced as well. This is because for many measurement, due to the mentioned improvements in the track impact-parameter resolution, the level of the combinatorial background will significantly be be reduced, and the feed-down contribution from beauty to charm measurements will be estimated directly, from the data themselves.

There are also several observables, like those involving the heavy-flavour baryons and the lowmomentum di-electrons, that were never accessible before, but will become well within the experimental reach thanks to the unique capabilities of the upgraded ALICE Inner Tracking System.

Table 1. Summary of the physics reach: minimum accessible $p_{\mathrm{T}}$ and relative statistical uncertainty in $\mathrm{Pb}-\mathrm{Pb}$ collisions for an integrated luminosity of $10 \mathrm{nb}^{-1}$. For heavy flavour, the statistical uncertainties are given at the maximum between $p_{\mathrm{T}}=2 \mathrm{GeV} / c$ and $p_{\mathrm{T}}^{\min }$. For elliptic flow measurements, the value of $v_{2}$ used to calculate the relative statistical uncertainty $\sigma_{v_{2}} / v_{2}$ is given in parenthesis. The programme up to Long Shutdown 2, with an integrated luminosity of $0.1 \mathrm{nb}^{-1}$ collected with minimum-bias trigger, is shown for comparison.

\begin{tabular}{|c|c|c|c|c|}
\hline \multirow[b]{2}{*}{ Observable } & \multicolumn{2}{|c|}{ Current, $0.1 \mathrm{nb}^{-1}$} & \multicolumn{2}{|c|}{ Upgrade, $10 \mathrm{nb}^{-1}$} \\
\hline & $\begin{array}{c}p_{\mathrm{T}}^{\min } \\
(\mathrm{GeV} / c)\end{array}$ & $\begin{array}{c}\text { statistical } \\
\text { uncertainty }\end{array}$ & $\begin{array}{c}p_{\mathrm{T}}^{\min } \\
(\mathrm{GeV} / c)\end{array}$ & $\begin{array}{l}\text { statistical } \\
\text { uncertainty }\end{array}$ \\
\hline \multicolumn{5}{|c|}{ Heavy Flavour } \\
\hline D meson $R_{\mathrm{AA}}$ & 1 & $10 \%$ & 0 & $0.3 \%$ \\
\hline $\mathrm{D}_{\mathrm{s}}$ meson $R_{\mathrm{AA}}$ & 4 & $15 \%$ & $<2$ & $3 \%$ \\
\hline D meson from B $R_{\mathrm{AA}}$ & 3 & $30 \%$ & 2 & $1 \%$ \\
\hline $\mathrm{J} / \psi$ from $\mathrm{B} R_{\mathrm{AA}}$ & 1.5 & $15 \%$ (pT-int.) & 1 & $5 \%$ \\
\hline $\mathrm{B}^{+}$yield & \multicolumn{2}{|c|}{ not accessible } & 2 & $10 \%$ \\
\hline$\Lambda_{\mathrm{c}} R_{\mathrm{AA}}$ & \multicolumn{2}{|c|}{ not accessible } & 2 & $15 \%$ \\
\hline$\Lambda_{\mathrm{c}} / \mathrm{D}^{0}$ ratio & \multicolumn{2}{|c|}{ not accessible } & 2 & $15 \%$ \\
\hline$\Lambda_{\mathrm{b}}$ yield & \multicolumn{2}{|c|}{ not accessible } & 7 & $20 \%$ \\
\hline D meson $v_{2}\left(v_{2}=0.2\right)$ & 1 & $10 \%$ & 0 & $0.2 \%$ \\
\hline $\mathrm{D}_{\mathrm{s}}$ meson $v_{2}\left(v_{2}=0.2\right)$ & \multicolumn{2}{|c|}{ not accessible } & $<2$ & $8 \%$ \\
\hline $\mathrm{D}$ from $\mathrm{B} v_{2}\left(v_{2}=0.05\right)$ & \multicolumn{2}{|c|}{ not accessible } & 2 & $8 \%$ \\
\hline $\mathrm{J} / \psi$ from B $v_{2}\left(v_{2}=0.05\right)$ & \multicolumn{2}{|c|}{ not accessible } & 1 & $60 \%$ \\
\hline$\Lambda_{\mathrm{c}} v_{2}\left(v_{2}=0.15\right)$ & \multicolumn{2}{|c|}{ not accessible } & 3 & $20 \%$ \\
\hline \multicolumn{5}{|c|}{ Di-electrons } \\
\hline Temperature (intermediate mass) & \multicolumn{2}{|c|}{ not accessible } & & $10 \%$ \\
\hline Elliptic flow $\left(v_{2}=0.1\right)[11]$ & \multicolumn{2}{|c|}{ not accessible } & & $10 \%$ \\
\hline Low-mass spectral function [11] & \multicolumn{2}{|c|}{ not accessible } & 0.3 & $20 \%$ \\
\hline \multicolumn{5}{|c|}{ Hypernuclei } \\
\hline${ }_{\Lambda}^{3} \mathrm{H}$ yield & 2 & $18 \%$ & 2 & $1.7 \%$ \\
\hline
\end{tabular}




\section{References}

[1] F. Carminati et al. [ALICE Collaboration], J. Phys. G 30, 1517 (2004).

[2] G. Alessandro et al. [ALICE Collaboration], J. Phys. G 32, 1295 (2006).

[3] K. Aamodt et al. [ALICE Collaboration], JINST 3, S08002 (2008)

[4] J. Adam et al. [ALICE Collaboration], JHEP 03, 081 (2016)

[5] B. Abelev et al. [ALICE Collaboration], Phys. Rev. Lett. 111, 102301 (2013)

[6] B. Abelev et al. [ALICE Collaboration], Phys. Rev. Lett. 111, 222301 (2013)

[7] J. Adam et al. [ALICE Collaboration], Phys. Rev. C 93, 034913 (2016)

[8] B. Abelev et al. [ALICE Collaboration], J. Phys. G 41, 087002 (2014).

[9] http://towerjazz.com

[10] ALICE Collaboration, CERN-LHCC-2015-006, (2015)

[11] ALICE Collaboration, CERN-LHCC-2012-012, LHCC-I-022, (2012) 